\title{
GMR
}

\section{Associations between HLA-A|B|DRB1 polymorphisms and risks of vulvar lichen sclerosus or squamous cell hyperplasia of the vulva}

\author{
G.L. Liu ${ }^{1 *}$, F.L. Cao ${ }^{2 *}$, M.Y. Zhao ${ }^{1}$, J. Shi ${ }^{1}$ and S.H. Liu ${ }^{1}$ \\ 1'Department of Gynecology, Hospital of Heilongjiang Chinese Medicine, \\ Harbin, Heilongjiang, China \\ ${ }^{2}$ Department of Hematology/Central Laboratory, \\ First Affiliated Hospital of Harbin Medical University, Harbin, Heilongjiang, China \\ ${ }^{*}$ These authors contributed equally to this study. \\ Corresponding author: G.L. Liu \\ Email: liuguilanmedsci@163.com \\ Genet. Mol. Res. X (X): XXX-XXX (2015) \\ Received May 18, 2015 \\ Accepted August 22, 2015 \\ Published December 7, 2015 \\ DOI http://dx.doi.org/10.4238/2015.December.7.8
}

\begin{abstract}
In this study, we aimed to explore the associations between $H L A-A|B| D R B 1$ polymorphisms and the risks of vulvar lichen sclerosus (VLS) or squamous cell hyperplasia of the vulva (SCHV) in Han Chinese women. We enrolled 76 Han Chinese women with VLS (Group A), 74 with SCHV (Group B), and 66 healthy women (control group) in this study. Polymerase chain reaction amplification with sequence specific primers (PCR-SSP) was used to determine HLA-AIBIDRB1 polymorphisms. Compared with the control group, $H L A-A^{*} 11,-B^{*} 15$, and $-D R B 1 * 12$ were present at a higher frequency in groups $A$ and $B$, while $H L A-B^{*} 13$ was present at a higher frequency in group $A$. Fewer women in group $A$ carried $H L A-A^{*} 31,-D R B 1^{*} 01$, and $-D R B 1^{*} 03$ genotypes and fewer women in group $B$ carried $H L A-B^{*} 40$ and $-D R B 1^{*} 03$ genotypes. Significant differences were found between group $B$ and the control group for $H L A-A^{*} 11,-B^{*} 15,-B^{*} 40$, and $-D R B 1^{*} 03$, and between group $A$ and the control group for $H L A-B^{*} 15$ and $-D R B 1^{*} 12$. The HLA-A*11, HLA-B*13, HLA-B*15, and HLA-DRB1*12
\end{abstract}


genotypes were associated with a higher risk of VLS, while the $H L A-A^{*} 31$, $H L A-D R B 1^{*} 01$, and $H L A-D R B 1^{*} 03$ genotypes were associated with a lower risk of VLS. In addition, carrying $H L A-A^{*} 11, H L A-B^{*} 15, H L A-B^{*} 35$, and $H L A-D R B 1^{*} 12$ genotypes, and carrying $H L A-B^{*} 40$ and $H L A-D R B 1{ }^{*} 03$ genotypes were found to be risk or protective factors for $\mathrm{SCHV}$, respectively.

Key words: HLA-A|BIDRB1; Polymorphisms; Vulvar lichen sclerosus; Squamous cell hyperplasia of the vulva

\section{INTRODUCTION}

Non-neoplastic epithelial disorders of the vulva, including vulvar lichen sclerosus (VLS) and squamous cell hyperplasia of the vulva (SCHV), are characterized by pigment changes and dysplasia of the vulvar skin and mucosa. They are also called white lesions of the vulva because the vulval skin and mucosa of the patient might appear white. Statistical analysis showed that in every 300 to 1000 women, 1 would be expected to have a non-neoplastic epithelial disorder of the vulva (Viana et al., 2011). VLS is mainly characterized by atrophy and thinning of the skin of the vulva and/or the crissum. One study showed that 1.7 percent of patients seen by gynecologists (Cooper et al., 2008). In addition, VLS might also progress to vulvar squamous cell carcinoma; the rate of developing cancer has been reported to be in the range of 3 to $6 \%$, and as high as $25 \%$. Numerous studies have demonstrated that a proportion of vulvar squamous cell carcinomas had progressed from VLS (Rolfe et al., 2003). In comparison, pruritus of the vulva is the main symptom of SCHV, which might in turn progress to malignant disease in from 2 to $4 \%$ of patients. Approximately $35 \%$ of vulvar dystrophies show squamous cell hyperplasia pathology (O'Connell et al., 2008). Several factors such as immunity, genetics, infections, and hormones might be involved in the pathogenesis of SCHV. However, the etiology of vulvar dystrophy remains unknown, and such diseases are considered common but refractory.

Some studies have suggested that an autoimmune mechanism is involved in VLS (Oyama et al., 2003); thus, marked attention has been paid to the relationship between VLS and autoimmunity. Several kinds of autoantibodies exist in many patients with VLS, the most common of which are antibodies against the nucleus, the thyroid, and against gastric parietal cells (Birenbaum and Young, 2007). Similar results were also found by Simpkin et al. (2007), who reported that $48 \%$ of patients with VLS were found to have autoantibodies. In recent years, the anti-ECM1 antibody has been found in approximately $75 \%$ of patients with VLS, suggesting that humoral autoimmunity might play a role in this disease (Edmonds et al., 2011).

The human leukocyte antigen (HLA) system represents the major histocompatibility complex (MHC) in humans. Several studies have indicated that HLA is involved in many human diseases, and people with certain HLA genotypes might be more susceptible or resistant to specific diseases.HLA has been demonstrated to be involved in the genetic susceptibility to and the development of more than 500 diseases including malignancies; however, the majority are autoimmune disorders In a study published in 2005, researchers reported that HLA was closely related to more than 10 diseases (Ghodke et al., 2005). An association has also been found between lichen atrophic sclerosus and $H L A-A w^{*} 31$ or $H L A-B^{*} 40$ (Holt and Darke, 2010). In another study, Purcell et al. (1996) were the first to investigate HLA genotypes among Caucasians with lichen sclerosus et atrophicus (LSA) from the United States, and found that carrying either HLA-A*29 or $H L A-B^{*} 44$ could greatly influence LSA and that carrying these two genotypes simultaneously 
also had a significant influence on the disease. Gao et al. (2005) investigated the distribution of alleles among patients with VLS using polymerase chain reaction (PCR), and discovered that the frequencies of the $D R B 1 * 12$ (DR12) genotype and the $D R B 1^{*} 12 / D Q B 1 * 0301 / 04 / 09 / 010$ haplotype were higher in patients compared with the control group. However, DRB1*0301/04 polymorphisms and the $D R B 1^{*} 0301 / 04 / D Q B 1^{*} 0201 / 02 / 03$ haplotype were less common in patients than in the control group. Another study conducted by Aslanian et al. (2006) investigated VLS in 3 different families, and reported that $H L A-B^{*} 15, B^{*} 57, C W^{*} 03, C W^{*} 07, C W^{*} 18, D R B 1^{*} 04, D R B 1^{*} 07$, and $D R B 4^{*}$ were associated with a risk of VLS. However, Purcell et al. (1996) found no significant association, although they found that the frequencies of $H L A-B^{*} 8$ and $D R^{*} 3$ were higher in patients.

Familial clustering of VLS has also been observed in several studies. The disease has been found to occur in sisters, mothers and daughters, and in twin sisters including both homozygotic and heterozygotic twins, suggesting that humans might have a genetic predisposition to VLS. However, no evidence has suggested the involvement of specific autosomes, sex-linked inheritance, or certain genes in the disease.

Therefore, VLS has been classified as both a gene-related immunological and a genetic disease. Numerous studies have been conducted to evaluate the association between $H L A$ and LS, and several international researchers have demonstrated that the association between HLA gene polymorphism and LS varies across different ethnicities or regions; however, no study has been conducted to investigate the association in a Chinese population. There has also been no study to date that has reported the association between HLA gene polymorphism and the risk of SCHV.

In the present study, we found that the $H L A-A * 11,-B * 15$, and $-D R B 1 * 12$ genotypes were associated with both VLS and SCHV in Han Chinese women. Among these genotypes, $H L A-A * 11$ was associated with a risk of SCHV and $H L A-B^{*} 12$ was closely associated with a risk of VLS, while $H L A-B^{*} 15$ was closely associated with both diseases. The associations between the $H L A-B^{*} 15$ and $H L A-D R B 1 * 12$ genotypes and the risk of VLS were consistent with those reported by previous studies. However, to date, no study has investigated the association between $H L A-A|B| D R B 1$ polymorphisms and the risk of SCHV. HLA-B*40 was also found to be a susceptible genotype for VLS in previous studies; however, no association was found between the $H L A-B^{*} 40$ genotype and VLS in the present study, whereas this genotype was instead suggested to be a strong protective factor for SCHV. Thus, the findings of the present study indicate that SCHV is also an immunological and genetically-related disease.

\section{MATERIAL AND METHODS}

\section{Patient selection and recruitment}

We included 231 patients in the present study who had been treated in one of the 5 hospitals (Heilongjiang Provincial Hospital of Traditional Chinese Medicine, the First and Second Affiliated Hospitals of Harebin Medical University, the First Affiliated Hospital of Traditional Chinese Medical University of Heilongjiang, and the 242nd Hospital of Harbin) between January 2003 and July 2011, and who also had complete medical records. Among these patients, there were 123 with VLS, 92 with SCHV, and 16 with both VLS and SCHV.

Patients with ongoing psychiatric disorders, those who could not undergo a physical evaluation, in-patients or out-patients who were in critical condition or who could not understand the questionnaires, and patients who did not complete the questionnaires were excluded from the present study. There were 150 patients with an age range of 21 to 59 years who were included in the 
present study. Among these, 76 (50.67\%) were in the VLS group (group A) and 74 (49.33\%) were in the SCHV group (group B). We included 66 healthy women as the control group (all 66 carried HLA genotype, as confirmed by the experimental results reported from the First Affiliated Hospitals of Harbin Medical University). The characteristics of the patients are displayed in Table 1.

\begin{tabular}{|c|c|c|c|c|}
\hline & Group A & Group B & Control group & $\mathrm{P}$ \\
\hline Age (years) & $38.53 \pm 10.37$ & $36.93 \pm 9.57$ & $40.30 \pm 11.72$ & $>0.05$ \\
\hline Menopause (yes:no) & $13: 63$ & $10: 64$ & 9:57 & $>0.05$ \\
\hline Course of disease (years) & $5.85 \pm 5.75$ & $4.91 \pm 4.61$ & $\mathrm{n} / \mathrm{a}$ & $>0.05$ \\
\hline Comorbidity & 0 & 0 & 0 & $>0.05$ \\
\hline Autoimmune & Healthy & Healthy & Healthy & $>0.05$ \\
\hline
\end{tabular}

$\mathrm{n} / \mathrm{a}=$ not applicable.

\section{Diagnostic criteria for VLS and SCHV}

VLS and SCHV were diagnosed according to the progenital skin classification criteria issued by the International Society for the Study of Vulvovaginal Disease and the International Society of Gynecological Pathologists in 1987.

\section{VLS}

The symptoms of VLS include pruritus in the skin of the lesions, pain during sex; some patients might also exhibit dysuria or dyspareunia. Signs of VLS include swelling and redness of the skin at the early stage, and the lesion can further progress to white, thin, dry, and chapped skin and mucosa with a loss of elasticity as the disease advances. There can be atrophy or even a reduction in the labium minus. The pathologic diagnostic criteria of VLS include epidermal keratosis and hair follicles embolized by keratin; thinning of the prickle cell layer and liquefaction degeneration of basal cells; decreased number of melanocytes; an epithelial angle that is blunted or that has disappeared; and homogenized appearance of the superficial dermis, with frequent findings of lymphocytes and serous cell infiltration in the dermis.

\section{SCHV}

The major symptom of SCHV is pruritus, which is more serious than in VLS. Signs including lesions involving the labium majus, nympholabial furrow, preputium clitoridis, and commissura labiorum posterior. In the early disease stage, the skin might appear dark red or pink, while hyperkeratotic skin might appear white.

\section{Patient inclusion criteria}

Patients were eligible for the study if they met the following inclusion criteria: 1) met the diagnostic criteria for VLS or SCHV; 2) were aged between 20 and 60 years; and 3) approved blood samples being taken and signed the consent form.

\section{Patient exclusion criteria}

A patient was excluded from the present study if she: 1) was diagnosed with vulvar 
intraepithelial neoplasia, carcinoma in situ, or invasive carcinoma; and 2) had other immune-related diseases, including connective tissue disease, thyroid disease, systemic lupus erythematosus, psoriasis, diabetes, vitiligo, tumor, or neurological or psychiatric disorders.

\section{Control group inclusion criteria}

A healthy subject was included in the present study if she met the following criteria: 1) volunteered to participate and signed the consent form; and 2) had none of the following diseases: connective tissue disease, thyroid disease, systemic lupus erythematosus, psoriasis, diabetes, vitiligo, tumor, or neurological or psychiatric disorders.

\section{Genotyping analysis}

\section{Genomic DNA extraction}

The patients and control subjects were asked to fast before blood collection. Peripheral venous blood (2.0 to $5.0 \mathrm{~mL}$ ) from each subject was anticoagulated using ethylenediaminetetraacetic acid. Leukocytes were extracted and stored $a t-20^{\circ} \mathrm{C}$. Human genomic DNA was extracted from whole blood using the DNeasy Blood \& Tissue Kit (Qiagen, Valencia, CA, USA) according to the manufacturer's instructions). The extracted DNA was then analyzed for purity and yield via absorbance spectroscopy (A260/A280) using the NanoDrop ND 2000 spectrophotometer (Thermo Fisher Scientific) and stored at $-70^{\circ} \mathrm{C}$. This mixture was stored at $-20^{\circ} \mathrm{C}$ until use. The DNA concentration was determined using a spectrophotometer. The DNA was diluted to a concentration of $50 \mathrm{mg} / \mathrm{mL}$ for further use.

\section{PCR with sequence-specific primers (PCR-SSP)}

PCR-SSP was used to determine patient HLA genotypes. In the present study, the primers were selected based on previous studies (Bunce and Passey, 2013) and purchased from One Lambda Company (Canoga Park, CA, USA). The final concentration of each primer used for the amplification of $H L A$ DNA was $10 \mu \mathrm{M}$, containing 20 pmol primer. The concentration of the primer internal control was $1 / 10$ that of the HLA DNA primer (2 pmol) (Tan et al., 1998). For PCR amplifications were performed in a $20-\mu \mathrm{L}$ reaction containing $5 \mu \mathrm{L} 25 \mathrm{ng}$ input DNA, $10 \mu \mathrm{L}$ 2X AmpliTaq Gold PCR Master Mix (Applied Biosystems, Foster City, CA, USA). The PCR was performed using a GeneAmp PCR System 9700 thermal cycler (Applied Biosystems) with the following cycling conditions: the DNA was first denatured at $94^{\circ} \mathrm{C}$ for $5.5 \mathrm{~min}$, then annealed at $60^{\circ} \mathrm{C}$ for $50 \mathrm{~s}$, and elongated at $72^{\circ} \mathrm{C}$ for $40 \mathrm{~s}$ for the first 5 cycles; then the annealing temperature was changed to $58^{\circ} \mathrm{C}$ while the other conditions were not changed for the remaining cycles. A total of 34 cycles were performed.

The PCR products were analyzed by agarose gel $(1.5 \%)$ electrophoresis at $12 \mathrm{mV} / \mathrm{cm}$, and then the DNA was labeled using Goldview ${ }^{\mathrm{TM}}$ nucleic acid stain (Newprobe, Peking, China) and observed and photographed under ultraviolet light.

\section{Statistical analysis}

All statistical analyses were performed using the SPSS 10.0 software package (SPSS, Chicago, IL, USA). HLA-AIBIDRB1 genotype frequencies were compared among each group and analyzed using a $X^{2}$ test. $P<0.05$ was considered to be statistically significant. 


\section{RESULTS}

\section{Distribution of $H L A-A * 11,-B * 13,-B * 15,-B * 35$, and $-D R B 1 * 12$}

Higher frequencies of $H L A-A^{*} 11,-B^{*} 13,-B^{*} 15$, and $-D R B 1 * 12$ genotypes were found in group $A$, while more women in group $B$ were found to carry $H L A-A^{*} 11,-B^{*} 15,-B^{*} 35$, or $-D R B 1^{*} 12$ genotypes. There were significant differences between the frequencies of $H L A-B^{*} 15(P<0.001)$, $-D R B 1 * 12(P<0.001),-A * 11(P<0.05)$, and $-B^{*} 13(P<0.05)$ in group $A$ compared with those in the control group. Significant differences were also detected when comparing the frequencies of $H L A-A * 11(P<0.001),-B * 15(P<0.001), H L A-B * 35(P<0.05)$, and $-D R B 1 * 12(P<0.05)$ genotypes between group $B$ and the control group.

\section{Distribution of $H L A-A^{*} 31,-B^{*} 40,-D R B 1^{*} 01$, and $-D R B 1^{*} 03$}

Significant differences were found between group $A$ and the control group when comparing the frequencies of the $H L A-A^{*} 31,-D R B 1^{*} 01$, and $-D R B 1^{*} 03$ genotypes $(\mathrm{P}<0.05)$; fewer women in group $A$ than in the control group were found to carry these genotypes. Similarly, fewer women in group $\mathrm{B}$ were found to carry the $H L A-B^{\star} 40$ or $-D R B 1^{*} 03$ genotypes compared with the control group $(\mathrm{P}<0.001)$ (Table 2$)$.

\section{DISCUSSION}

HLA has the widest range of human genetic polymorphisms, and these polymorphisms are responsible for ethnic, race, and regional differences. It has been demonstrated that the HLA system, and especially $H L A-A|B| D R B 1$ polymorphisms, accurately regulate the global immunity of humans and thus play critical roles in immune reactions. In addition, these polymorphisms play key roles in mediating immune responses. Every human cell has its own HLA identity, which can help to distinguish "self cells" and remove "non-self cells" to maintain the body's integrity. Autoimmune diseases such as VLS might occur when this process becomes abnormal.

The results of the present study showed that the $H L A-A * 11,-B * 15$, and $-D R B 1 * 12$ genotypes were associated with the risk of VLS, and also influenced the susceptibility to SCHV. The HLA-A*11 and DRB1*12 genotypes appeared to be closely related to higher risks of SCHV and VLS, respectively, while the HLA-B*15 genotype was found to be closely related to a higher risk of VLS and of SCHV. HLA-A*11, and $-B^{*} 13$ genotypes were also associated with a higher risk of VLS, and $H L A-B^{*} 35$ and $-D R B 1 * 12$ genotypes were associated with a higher risk of SCHV, although the associations were not strong. The results further suggested that the $H L A-D R B 1^{*} 03$ and $-B^{*} 40$ genotypes were strong protective factors for $\mathrm{SCHV}$, and that the $H L A-A^{*} 31,-D R B 1^{*} 01$, and $-D R B 1^{*} 03$ genotypes were associated with a lower risk of VLS. In addition, the HLA-DRB ${ }^{*} 03$ genotype seemed to be a strong protective factor for both VLS and SCHV.

The present study found that carrying an $H L A-A^{*} 11,-A^{*} 31,-B^{*} 13,-B^{*} 15,-D R B 1^{*} 01$, $-D R B 1^{*} 03$, or $-D R B 1^{*} 12$ genotype might influence the susceptibility to VLS, whereas carrying a $H L A-A^{*} 11,-B^{*} 15,-B^{*} 35,-B^{*} 40,-D R B 1^{*} 03$, or $-D R B 1^{*} 12$ genotype might influence the susceptibility to SCHV. The risk of developing VLS decreases in those carrying the $H L A-A * 31,-D R B 1^{*} 01$, or $-D R B 1^{*} 03$ genotypes, greatly increases in those carrying the $H L A-B^{*} 15$ or $H L A-D R B 1^{*} 12$ genotypes, and moderately increases in those carrying the $H L A-A{ }^{*} 11$ or $-B^{*} 13$ genotypes. The 
risk of developing SCHV might be greatly increased in those carrying the $H L A-A^{*} 11$ or $-B^{*} 35$ genotypes, greatly decreased in those carrying the $H L A-B^{*} 40$ or $-D R B 1^{*} 03$ genotypes, and moderately increased in those carrying the $H L A-B^{*} 35$ or $-D R B 1^{*} 12$ genotypes.

\begin{tabular}{|c|c|c|c|c|c|}
\hline $\begin{array}{l}\text { HLA-AI } \\
B \mid D R B 1\end{array}$ & $\begin{array}{l}\text { Group A } \\
(\mathrm{N}=76)\end{array}$ & $\begin{array}{l}\text { Group B } \\
(\mathrm{N}=74)\end{array}$ & $\begin{array}{l}\text { Control Group } \\
\qquad(\mathrm{N}=66)\end{array}$ & $\begin{array}{c}\text { OR } \\
\text { (Group A) }\end{array}$ & $\begin{array}{c}\text { OR } \\
\text { (Group B) }\end{array}$ \\
\hline A01 & 5 & 12 & 12 & 0.34 & 0.88 \\
\hline A02 & 78 & 48 & 58 & 1.34 & 0.61 \\
\hline $\mathrm{A} 03$ & 6 & 10 & 6 & 0.86 & 1.52 \\
\hline A11 & $24^{*}$ & $32^{\star \star \star}$ & 8 & 2.90 & 4.28 \\
\hline A24 & 23 & 24 & 10 & 2.17 & 2.36 \\
\hline A30 & 2 & 4 & 4 & 0.43 & 0.89 \\
\hline A31 & $2^{*}$ & 8 & 10 & 0.16 & 0.70 \\
\hline A32 & 0 & 4 & 6 & 0 & 0.58 \\
\hline A33 & 7 & 2 & 6 & 1.01 & 0.29 \\
\hline A68 & 2 & 0 & 6 & 0.28 & 0 \\
\hline B07 & 2 & 2 & 2 & 0.87 & 0.89 \\
\hline B08 & 0 & 4 & 2 & 0 & 1.80 \\
\hline B13 & $29^{*}$ & 20 & 12 & 2.36 & 1.56 \\
\hline B15 & $35^{* \star *}$ & $28^{\star \star *}$ & 6 & 6.28 & 4.90 \\
\hline B18 & 1 & 2 & 0 & 0.48 & 0 \\
\hline B27 & 6 & 4 & 6 & 0.86 & 0.58 \\
\hline B35 & 9 & $14^{*}$ & 4 & 2.01 & 3.34 \\
\hline B37 & 0 & 2 & 0 & 0 & 0 \\
\hline B38 & 0 & 2 & 0 & 0 & 0 \\
\hline B40 & 19 & $8^{\star \star \star}$ & 30 & 0.49 & 0.19 \\
\hline B44 & 9 & 4 & 8 & 0.96 & 0.43 \\
\hline B46 & 5 & 2 & 4 & 1.09 & 0.44 \\
\hline B48 & 6 & 2 & 4 & 1.52 & 0.44 \\
\hline B49 & 0 & 2 & 0 & 0 & 0 \\
\hline B50 & 2 & 0 & 0 & 0 & 0 \\
\hline B51 & 10 & 18 & 10 & 0.86 & 1.69 \\
\hline B52 & 0 & 6 & 2 & 0 & 2.75 \\
\hline B54 & 2 & 12 & 6 & 0.28 & 1.85 \\
\hline B55 & 2 & 4 & 0 & 0 & 0 \\
\hline B56 & 1 & 0 & 2 & 0.43 & 0 \\
\hline B57 & 2 & 4 & 6 & 0.28 & 0.58 \\
\hline B58 & 3 & 4 & 4 & 0.64 & 0.89 \\
\hline B67 & 3 & 2 & 0 & 0 & 0 \\
\hline DR01 & $0^{*}$ & 6 & 6 & 0 & 0.89 \\
\hline DR03 & $2^{\star *}$ & $0^{\star \star *}$ & 12 & 0.13 & 0 \\
\hline DR04 & 13 & 16 & 16 & 0.68 & 0.88 \\
\hline DR07 & 13 & 10 & 16 & 0.68 & 0.53 \\
\hline DR08 & 2 & 6 & 0 & 0 & 0 \\
\hline DR09 & 16 & 28 & 12 & 1.18 & 2.33 \\
\hline DR10 & 0 & 2 & 2 & 0 & 0.89 \\
\hline DR11 & 8 & 8 & 10 & 0.68 & 0.70 \\
\hline DR12 & $77^{\star \star \star}$ & $30^{*}$ & 12 & 10.27 & 2.54 \\
\hline DR13 & 2 & 10 & 12 & 0.13 & 0.72 \\
\hline DR14 & 6 & 10 & 8 & 0.64 & 1.12 \\
\hline DR15 & 12 & 14 & 16 & 0.62 & 0.76 \\
\hline DR16 & 1 & 2 & 2 & 0.43 & 0.89 \\
\hline
\end{tabular}

${ }^{*} \mathrm{P}<0.05 ;{ }^{* *} \mathrm{P}<0.01 ;{ }^{* * *} \mathrm{P}<0.001$. VLS = vulvar lichen sclerosus; $\mathrm{SCHV}=$ squamous cell hyperplasia of the vulva.

The present study also indicated that women who carry the $H L A-A^{*} 11,-B^{*} 15$, or $-D R B 1^{*} 12$ genotype might be at an increased risk of developing VLS and SCHV simultaneously, whereas carrying the $H L A-D R B 1{ }^{*} 03$ genotype might decrease the risk of each of these diseases. HLA-A*31, and $-B^{*} 13$ are among the primary VLS susceptibility polymorphisms, while $H L A-D R B 1{ }^{*} 01$ is a main protective polymorphism. Similarly, for SCHV, $H L A-B^{*} 35$ is a primary susceptibility polymorphism, and $H L A-B^{*} 40$ is a main protective polymorphism. 
As described above, Aslanian et al. (2006) found that several genotypes including $H L A-B^{*} 15$ were associated with VLS. This is consistent with our findings, which demonstrated that the $H L A-B^{*} 15$ genotype was closely associated with the risk of developing VLS in Han Chinese women. The present study also showed, for the first time, that the $H L A-B^{*} 15$ genotype was also closely related to SCHV in Han Chinese women.

Studies have found that the MHC-DRB*12 genotype is more frequent in patients with VLS compared with healthy women. These patients also appear to be sensitive to vulvar dystrophy treatment, which might be mechanistically related. In the present study, the HLA-DRB1*12 genotype was found to be closely associated with VLS susceptibility, which was consistent with other studies. Additionally, this study showed for the first time that the HLA-DRB1*12 genotype was associated with SCHV susceptibility.

Research has also found that patients with VLS have a higher frequency of carrying the $H L A-B^{*} 40$ genotype. However, in the present study, we did not detect increased frequencies of the $H L A-B * 40, H L A-B * 44$, or $H L A-B^{*} 57$ genotypes. In contrast, the $H L A-B * 40$ genotype appeared to have a strong protective effect against SCHV in Han Chinese women, because the frequency of this genotype was observed to be decreased in patients with SCHV. Similarly, the frequency of the $H L A-B * 40$ genotype was lower in Han Chinese women with VLS, but no significant difference was found. The difference between our results and those from the previous studies suggest that the association varies in different ethnicities or regions, and that even in patients with the same disease, the underlying genetic polymorphisms might vary broadly. A relationship between $H L A-$ $A|B| D R B 1$ polymorphism and the risk of SCHV has not been previously reported; this is the first study suggesting that a relationship exists.

The findings of the present study demonstrated that there are some common susceptibility genes, such as $H L A-B^{*} 15$ and $-D R B 1^{*} 12$, between Han Chinese women and women in other countries who have VLS. We also identified several other susceptibility genes (including $H L A-A^{*} 11$ and $H L A-B^{*} 13$ ) and protective genes (including $H L A-D R^{*} 03,-D R^{*} 01$, and $A^{*} 31$ ) in Han Chinese women. In addition, an association between $H L A$ gene polymorphisms and the risk of SCHV in Han Chinese women was also found. Several genes, including $H L A-B * 15,-D R B 1 * 12$, and $-A * 11$, were shown to be susceptibility genes, and -DRB1*03 was shown to be a protective gene, for both VLS and SCHV. In contrast, $H L A-B * 35$ and $H L A-B * 40$ were shown to be susceptibility and protective genes for SCHV, respectively, but not for VLS. The findings of the present study suggest that SCHV could also be a genetic immune disease, and our study further provides evidence that VLS and SCHV are related nonneoplastic epithelial disorders of the vulva.

To our knowledge, among the more than 500 diseases that are associated with HLA, strong associations are found in only 10 diseases including ankylosing spondylitis, insulin dependent diabetes mellitus, Goodpasture's syndrome, pemphigus vulgaris, coeliac disease, systemic lupus erythematosus, rheumatoid arthritis, and multiple sclerosis. The findings of the present study demonstrated that VLS and SCHV are also genetic immune diseases that are strongly associated with HLA.

We hypothesize that, in the near future, analysis of HLA genotypes and screening of susceptibility genes and/or resistance genes might be used in practice to identify populations that are susceptible to VLS and SCHV. We believe that investigating the status of $H L A-B^{*} 15, H L A$ $D R * 12, H L A-B * 40, H L A-A * 11, H L A-A * 31, H L A-B * 13, H L A-B * 35, H L A-D R B 1 * 01$, or HLA-DRB1*03 genotypes in patients with VLS or SCHV might aid in the early diagnosis of these diseases, and also contribute to their prediction, prevention, and treatment. 
Several studies have demonstrated that the development of VLS and SCHV is affected by multiple factors and is associated with immunity, sexual hormones, injuries, environment, enzymes, free radicals, and apoptosis. However, the present study was limited by its focus on the investigation of the association between HLA gene polymorphisms and VLS or SCHV, whereas the other risk factors were not included in our analysis. In addition, the present study had a relatively small sample size. Multi-center studies with larger sample sizes are required to validate our findings, to further investigate the relationship between low frequency variants and haplotypes, and to perform pedigree research to investigate the risk of white lesions of the vulva, and the contribution of environmental and other factors therein.

\section{ACKNOWLEDGMENTS}

Thanks are due to the Department of Hematology, the First Affiliated Hospital of Harbin Medical University for technical assistance to the experiments and to the Heilongjiang Provincial Hospital of Traditional Chinese Medicine, the First Affiliated Hospital of Harbin Medical University, the Second Affiliated Hospital of Harbin Medical University, and the 242nd Hopital of Harbin for collecting the cases.

\section{Conflicts of interest}

The authors declare no conflict of interest.

\section{REFERENCES}

Aslanian FM, Marques MT, Matos HJ, Pontes LF, et al. (2006). HLA markers in familial lichen sclerosus. J. Dtsch. Dermatol. Ges. 4: 842-847.

Birenbaum DL and Young RC (2007). High prevalence of thyroid disease in patients with lichen sclerosus. J. Reprod. Med. 52: 28-30.

Bunce M and Passey B (2013 ). HLA typing by sequence-specific primers. Meth. Mol. Biol. 1034: 147-159.

Cooper SM, Ali I, Baldo M and Wojnarowska F (2008). The association of lichen sclerosus and erosive lichen planus of the vulva with autoimmune disease: a case-control study. Arch. Dermatol. 144: 1432-1435.

Edmonds EV, Oyama N, Chan I, Francis N, et al. (2011). Extracellular matrix protein 1 autoantibodies in male genital lichen sclerosus. Br. J. Dermatol. 165: 218-219.

Gao XH, Barnardo MC, Winsey S, Ahmad T, et al. (2005). The association between HLA DR, DQ antigens, and vulval lichen sclerosus in the UK: HLA DRB112 and its associated DRB112/DQB10301/04/09/010 haplotype confers susceptibility to vulval lichen sclerosus, and HLA DRB10301/04 and its associated DRB10301/04/DQB10201/02/03 haplotype protects from vulval lichen sclerosus. J. Invest. Dermatol. 125: 895-899.

Ghodke Y, Joshi K, Chopra A and Patwardhan B (2005). HLA and disease. Eur. J. Epidemiol. 20: 475-488.

Holt PJA and Darke C (1983). HLA antigens and Bf allotypes in lichen sclerosus et atrophicus. Tissue Antigens 22: 89-91.

O'Connell TX, Nathan LS, Satmary WA and Goldstein AT (2008). Non-neoplastic epithelial disorders of the vulva. Am. Fam. Physician 77: 321-326.

Oyama N, Chan I, Neill SM, Hamada T, et al. (2003). Autoantibodies to extracellular matrix protein 1 in lichen sclerosus. Lancet 362: 118-123.

Purcell KG, Spencer LV, Simpson PM, et al. (1996). HLA antigens in lichen sclerosus et atrophicus. Arch. Dermatol. 126: 1043-1045.

Rolfe KJ, MacLean AB, Crow JC, Benjamin E, et al. (2003). TP53 mutations in vulval lichen sclerosus adjacent to squamous cell carcinoma of the vulva. Br. J. Cancer 89: 2249-2253.

Simpkin S and Oakley A (2007). Clinical review of 202 patients with vulval lichen sclerosus: a possible association with psoriasis. Australas. J. Dermatol. 48: 28-31. 
Tan J, Tang X and Xie T (1998). DNA typing for HLA-A, B antigens by polymerase chain reaction with sequence-specific primers and clinical application. Zhonghua Yi Xue Za Zhi 78: 763-767.

Viana F de O, Cavaleiro LH, Unger DA, Miranda MF, et al. (2011). Acral lichen sclerosus et atrophicus-Case report. An. Bras. Dermatol. 86: 82-84. 